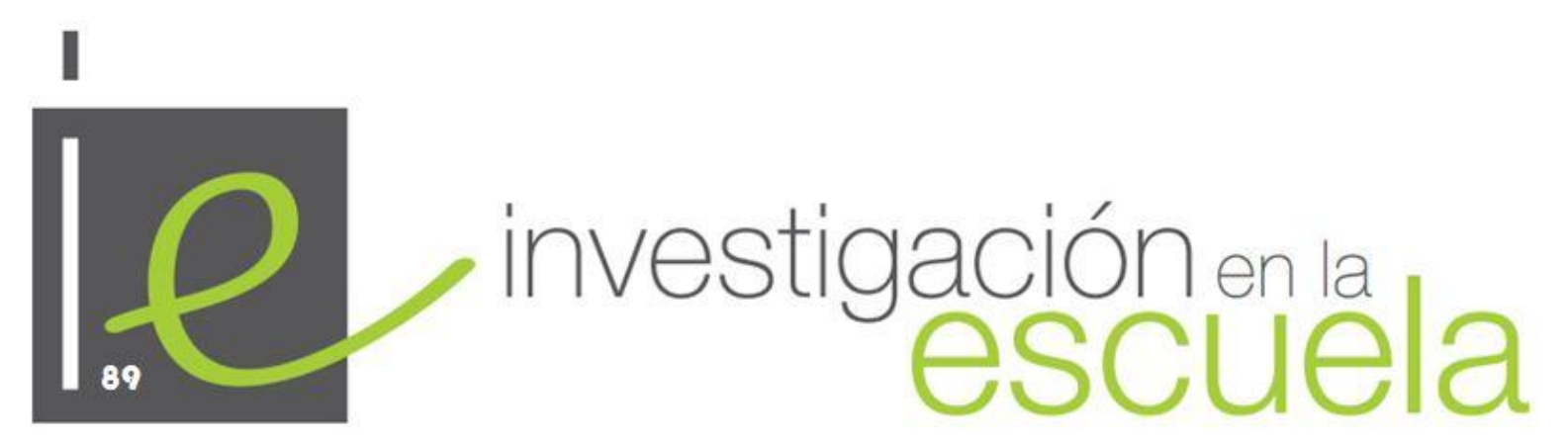

Revista académica evaluada por pares y de acceso abierto

\title{
El patrimonio como instrumento para la formación de la ciudadanía crítica y participativa
}

\author{
Laura Lucas Palacios y Jesús Estepa Giménez? \\ Universidad de Huelva \\ España
}

Citación: Lucas, L. \& Estepa, J. (2016). El patrimonio como instrumento para la formación de la ciudadanía crítica y participativa. Investigación en la Escuela, 89, 35-48. Recuperado de: http://www.investigacionenlaescuela.es/articulos/R89/R89-3.pdf

Resumen: Presentamos los resultados de una investigación llevada a cabo con el fin de detectar las concepciones que maneja el profesorado de Ciencias Sociales de la ESO en relación con el Patrimonio como contenido para la formación de una ciudadanía crítica, responsable y participativa y contrastarlo con el pensamiento del alumnado al que le da clase. El instrumento de recogida de información utilizado fue un cuestionario, diseñado en base a una tabla de categorías que, a su vez, se utilizó para el análisis de los datos.

Palabras clave: "Educación Patrimonial"; "concepciones sobre patrimonio"; "concepciones sobre ciudadanía"; "educación ciudadana”.

Heritage as an instrument for the formation of a critical citizenship and participatory Abstract: We present the results of an investigation carried out in order to detect the conceptions that handles faculty of Social Sciences ESO regarding the Heritage as content for the formation of a

\footnotetext{
${ }^{11}$ Universidad de Huelva, miembro del Grupo de Investigación DESYM y de la Red IRES.
} 
critical, responsible and participatory citizenship and contrast it with the thought of that gives students the class. The data collection instrument was a questionnaire, designed based on a table of categories that, in turn, was used for data analysis.

Key words: "Heritage Education"; "conceptions heritage"; "concepts of citizenship"; "civic education".

\section{Le patrimoine comme un instrument pour la formation d'une citoyenneté critique et participative.}

Résumé: Nous présentons les résultats d'une enquête menée dans le but de détecter les conceptions qui gère la faculté des sciences sociales de l'ESO concernant le patrimoine comme contenu pour la formation d'une citoyenneté critique, responsable et participative et le contraste avec la pensée de qui donne aux étudiants la classe. L'instrument de collecte de données était un questionnaire, conçu sur la base d'une table de catégories qui, à son tour, a été utilisé pour l'analyse des données. Mosts clé: "éducation au patrimoine"; "le patrimoine conceptions"; "concepts de citoyenneté"; "l'éducation civique".

\section{Introducción y Justificación}

La globalización y la multiculturalidad son un hecho, haciéndonos descubrir una nueva ciudadanía que hace visible la pluralidad de identidades, la diversidad y la alteridad como características básicas de las sociedades actuales. Siguiendo a Audiguier (1996), no formamos a nuestros jóvenes para responder a las preguntas de ayer, sino a aquellas que tendrán que responder mañana, de manera que estas respuestas respeten un conjunto de principios y valores alrededor de una ciudadanía activa y democrática. Partiendo de estos presupuestos, consideramos que la participación ciudadana es la clave para afrontar los problemas de un mundo en constante cambio, de manera que a través de la acción y el compromiso social, la ciudadanía colabore actuando localmente en la resolución de los problemas globales. Por ello, entendemos que la educación ciudadana se convierte en uno de los objetivos a incidir en la necesaria y compleja educación en valores que, mediante el conocimiento y la valoración del patrimonio, pueden potenciarse.

Las potencialidades educativas que tiene el patrimonio apuntan a una formación integral. Las propuestas de conexión entre educación patrimonial y participación ciudadana se hacen en torno a lo que Cuenca (2010) ha denominado la socialización del patrimonio. Se trataría de que los alumnos puedan "socializar el patrimonio" (Cuenca y Martín, 2009), es decir, conectar el hecho patrimonial con su realidad como jóvenes y con la sociedad en general, con el fin de difuminar la frontera entre los contenidos históricos y geográficos, para explicar una realidad a través de contenidos patrimoniales (Estepa y Morón, 2013). En definitiva, se trata de trabajar lo social a partir de lo patrimonial, defendiendo un modelo de enseñanza y aprendizaje que emplee el patrimonio como eje constructor de los contenidos de Ciencias Sociales.

El conocimiento del patrimonio implica tanto el desarrollo de procedimientos y valores individuales, como la autonomía o el juicio crítico; como de aquellos que tienen incidencia en el ámbito comunitario, como la tolerancia, el respeto o la solidaridad. Además, el patrimonio ayuda a la comprensión del mundo tanto en procedimientos como en recursos, basándose en las posibilidades de intervención y participación directa que tienen los ciudadanos sobre él. La ciudadanía puede intervenir de forma dinámica y sentirse partícipe del patrimonio mediante la gestión y conservación del mismo. 


\section{El Patrimonio como recurso para formar ciudadanos}

Tal y como acabamos de defender en el apartado anterior, la educación para la ciudadanía la entendemos como el conjunto de conocimientos y competencias que permiten al individuo explorar y comprender la realidad en toda su complejidad y actuar sobre ella de forma crítica, responsable y solidaria, de acuerdo con los valores democráticos. Por otra parte, la educación patrimonial en su actual acepción no tiene sentido sin el ciudadano: heredero de ese legado y responsable de su transmisión a las generaciones futuras; sujeto activo que participa en la defensa y mejora del medio ambiente, así como en el disfrute y valoración de estos bienes como señas de identidad, sin menoscabo del respeto y valoración de los patrimonios generados en otras culturas (Estepa, Wamba y Jiménez, 2005).

La propia etimología del término patrimonio remite a la ciudadanía al hacer referencia a la propiedad de los bienes recibidos por una comunidad de sus antepasados. De este modo, el patrimonio se configura como una herencia pública o como patrimonio colectivo, que debe ser interpretado por los ciudadanos para identificarse con él y debe ser preservado para las generaciones futuras, en un contexto social caracterizado por un modelo de desarrollo insostenible, el consumo indiscriminado y la homogeneización de las costumbres. Es por ello, por lo que defendemos que identidad, conservación y gestión se convierten en tres conceptos claves para el análisis de las relaciones entre educación patrimonial y educación para la ciudadanía (Estepa, 2009).

En lo referente a la identidad, cuando alguien posee algo, aunque sea colectivamente, lo identifica como propio, por lo que los ciudadanos deben ser quienes seleccionen los bienes patrimoniales como símbolo de su identidad colectiva para que sean reflejo de una cultura compartida en la que se reconocen las personas como miembros de esa comunidad, con sus raíces en el pasado, con sus realidades actuales y con sus proyectos de futuro.

Sin embargo, como constatan Ávila y Mattozzi (2009), debido a la vigencia de un modelo didáctico tradicional (Ávila, 2001; Estepa y Ávila, 2007), la enseñanza del patrimonio está caracterizada por una selección del conocimiento ligada al patrimonio histórico-artístico y a la enseñanza de la Historia del Arte, influenciada por la institución universitaria. Desde este modelo se forma a una ciudadanía pasiva que contempla al patrimonio como obra de arte, por su significado de grandiosidad y belleza artística y estilística. La cultura patrimonial con la que se forman los jóvenes es de tipo academicista, ya que su finalidad básica es el conocimiento de hechos e informaciones de carácter cultural, ilustrado y/o centrado en aspectos anecdóticos, a veces muy ligado a una finalidad práctica-conservacionista, vinculada al valor económico y/o sentimental que el elemento patrimonial tiene en la vida cotidiana de los individuos (Ávila y Mattozzi, 2009).

Se recurre así a una enseñanza del patrimonio muy relacionada con escalas de identidad individual, ya que sólo se reconocen aquellos elementos patrimoniales cercanos y vinculados con la experiencia personal del individuo. Además, la identificación de lo patrimonial que hacen muchos profesores de Ciencias Sociales, Geografía e Historia, está relacionada con la existencia de una imagen, esto lleva consigo que el patrimonio se considere concreto y tangible, asociado, como indican Estepa y Morón (2013), a la "trilogía clásica" (patrimonio natural, histórico y artístico).

Además, defendemos una didáctica del patrimonio que persiga una visión intercultural, en la que el reconocimiento del valor identitario del patrimonio traspasa las fronteras culturales, pudiendo alcanzar una consideración multi-identitaria a través de los referentes patrimoniales (Domínguez y Cuenca, 2005).

En cuanto a la gestión del hecho patrimonial, es evidente que para que los ciudadanos puedan hacer una buena gestión del patrimonio, deben sentirse herederos y propietarios de él. En este sentido, Ávila (2005) propone que el estudiante lo vincule a su experiencia vital para que se interese y quiera conocerlo. Para que el alumnado sea consciente de sus derechos y deberes en 
relación con el patrimonio, necesita no sólo adquirir una serie de instrumentos para su lectura, sino también verse definido como un agente social que pueda intervenir en su defensa y conservación (Estepa, 2009) y ser conscientes de la realidad en la que este patrimonio está presente.

Por ello, es necesario enseñar al alumnado a participar en la toma de decisiones y en la gestión del patrimonio, para que sea capaz de analizar, valorar y contrastar distintos puntos de vista, diferenciando el valor de cada uno de ellos y desarrollando actitudes acordes con la importancia de la preservación del mismo (Estepa, 2009). Ávila y Duarte (2012) señalan que es necesario evidenciar el valor social del estudio del patrimonio, su utilidad didáctica como organizador de los contenidos en base a problemáticas presentes y como estrategia para formar a un alumnado crítico y comprometido con su realidad.

Por todo ello, la educación patrimonial se configura como una praxis educativa y social que permite elaborar acciones pedagógicas que pretenden algo más que un estudio del pasado, abordando temas del currículum básico que atraviesan la educación ambiental y ciudadana. De este modo, se favorecen las economías locales a través del desarrollo turístico y de sostenibilidad y además el sentimiento de pertenencia y los lazos afectivos entre los miembros de una comunidad (Texeira, 2006).

A pesar de la directa relación entre patrimonio y ciudadanía, la contribución de la educación patrimonial a la educación ciudadana es todavía poco relevante en la educación formal, quedando reducida en la ESO, según Ávila (2005), a ilustraciones en los libros de texto, y en el Bachillerato, a ser una materia exclusivamente de la especialidad de Humanidades y Artística. Si bien es cierto que en los últimos años se han realizado una serie de investigaciones que ponen de manifiesto algunos logros conseguidos (Ávila y Duarte, 2012; Gil-Díez, 2012; Martín, Cuenca y Bedia, 2012).

Queda así expuesta la estrecha relación que establecemos entre educación patrimonial y educación para la participación ciudadana. Conocer qué concepciones y conocimiento del patrimonio tiene el profesorado en activo y ver si incluye el patrimonio con una integración plena en el currículum educativo y si traslada y ayuda a su alumnado a desarrollar valores identitarios, de uso y de participación, es una problemática importante que hay que abordar desde el ámbito de la investigación didáctica y es básicamente lo que nos proponemos con este trabajo.

\section{Metodología}

Teniendo en cuenta lo anterior, el objetivo de esta investigación es detectar las concepciones que maneja el profesorado de la ESO en relación con el patrimonio como contenido pedagógico en la materia de Ciencias Sociales para la formación de una ciudadanía crítica, responsable y participativa y contrastarlo con el pensamiento del alumnado al que le da clase. De este objetivo de investigación se desprenden los siguientes subproblemas:

1. ¿Qué entiende el profesorado de Ciencias Sociales por patrimonio? ¿Qué valoración educativa le da?

2. ¿Qué entiende el profesorado de Ciencias Sociales por educación para la ciudadanía?

3. ¿Cómo conectar patrimonio y ciudadanía en el proceso de enseñanza y aprendizaje y para qué?

4. ¿Qué aprenden los alumnos en clase sobre patrimonio y ciudadanía? ¿Son trasladadas las concepciones de sus profesores a este respecto en la asignatura de Ciencias Sociales?

5. ¿Creen los alumnos que pueden aprender a ser ciudadanos críticos y participativos a través del conocimiento del Patrimonio que reciben en sus clases? 
A fin de obtener el máximo de información posible que nos condujera a encontrar respuestas a nuestras preguntas de investigación, hemos utilizado el cuestionario como instrumento de recogida de datos. Según Rodríguez, Gil y García (1996), el cuestionario "permite abordar los problemas desde una óptica exploratoria, no en profundidad". No obstante, nuestra investigación se basa en un enfoque cuantitativo/cualitativo, pues combinamos el tratamiento estadístico de las respuestas con el análisis del contenido de las mismas, como indicador de las características y evolución de las concepciones del profesorado y del alumnado.Ambos cuestionarios se componen de 10 preguntas. En el caso del profesorado, 4 son abiertas y las 6 restantes de escala de valoración. El del alumnado tiene 5 preguntas abiertas, 1 de tipo dicotómico, 3 de opción múltiple y 1 cerrada. En ambos cuestionarios consideramos que dominan las preguntas de tipo abierto, con el fin de no inducir a respuestas que no reflejen las propias creencias de los encuestados. También hemos considerado conveniente utilizar preguntas de tipo dicotómico, que obligaran a los alumnos a posicionarse ante una cuestión, ya que es interesante analizar el argumento de dicho posicionamiento. Para preguntas más complejas, hemos empleado respuestas de opción múltiple.

No obstante, somos conscientes de las limitaciones de nuestro estudio, pues se trata de un ensayo investigativo que forma parte de un trabajo fin de máster, orientado a una investigación en mayor profundidad en una futura tesis doctoral. Por ello, las personas elegidas para el estudio son 9, profesores de Educación Secundaria, de los que hemos clasificado sus respuestas conforme a grupos de concepciones semejantes y seleccionar dos personas para una investigación futura en mayor profundidad, que nos permita conocer la conexión real entre lo que dice y lo que hace el profesorado.

El profesorado participante en nuestra investigación no responde a ningún criterio específico en cuanto a edad, experiencia, residencia, etc. Los únicos requisitos han sido ser profesor de Ciencias Sociales de Educación Secundaria Obligatoria en activo durante el curso en el que se aplicó el cuestionario y, en el caso de que lo consideremos oportuno, estar dispuestos a colaborar en un estudio de caso futuro. Además, al alumnado que participa en la investigación le imparte clase ese mismo profesorado, con el fin de analizar posibles relaciones entre las concepciones de unos y de otros. Hemos considerado que el número de alumnos que participa en la realización del cuestionario sea entre 7 a 10 por profesor, seleccionados por los propios docentes en los grupos-clase de $1^{\circ}, 2^{\circ}$, $3^{\circ}$ o $4^{\circ}$ de ESO, en total fueron 82 alumnos los encuestados.

Los datos obtenidos se han analizado usando un sistema de categorías y subcategorías definido por una serie de indicadores y descriptores. La estructuración y determinación de las categorías de análisis, así como de sus subcategorías y descriptores, es de gran importancia, ya que todas ellas se van a articular como el hilo conductor del proceso de indagación que se va a desarrollar, clarificando cual es el objeto de estudio y qué información se está tratando. Se plantearon tres categorías: a) concepciones sobre el patrimonio, b) concepciones acerca de ciudadanía, y c) concepciones sobre la enseñanza/aprendizaje de la ciudadanía a través del patrimonio. En la tabla 1 se describen las categorías y subcategorías, mediante indicadores y descriptores, indicando las preguntas del cuestionario que formulamos para obtener información sobre ellas, siguiendo los presupuestos teóricos y metodológicos empleados en otros trabajos (Estepa, Ávila y Ferreras, 2008; Estepa, Ferreras y Monge, 2013; Travé y otros, 2013). 
Tabla 1.

Sistema de categorías y preguntas de los cuestionarios del profesorado (P) y alumnado (A) relacionadas.

\begin{tabular}{|c|c|c|c|c|}
\hline CATEGORÍAS & SUBCATEG. & CUES & INDICADORES & DESCRIPTORES \\
\hline \multirow{11}{*}{ CATEGORÍA. I } & \multirow{5}{*}{$\begin{array}{l}\text { Concepto de } \\
\text { patrimonio }\end{array}$} & \multirow{3}{*}{$1 \mathrm{P} 2 \mathrm{P}$} & $\begin{array}{l}\text { Perspectiva } \\
\text { Excepcionalista }\end{array}$ & Escasez, rareza, singularidad. \\
\hline & & & $\begin{array}{l}\text { Perspectiva } \\
\text { Monumentalista }\end{array}$ & $\begin{array}{l}\text { Espectacularidad, reconocido prestigio, grandes } \\
\text { dimensiones }\end{array}$ \\
\hline & & & Persp. Estética & Belleza artística y estilística \\
\hline & & \multirow[b]{2}{*}{$1 \mathrm{~A} 2 \mathrm{~A}$} & Persp. Histórica & Antigüedad \\
\hline & & & $\begin{array}{l}\text { Persp. Simbólico- } \\
\text { identitaria }\end{array}$ & $\begin{array}{l}\text { Reconocimiento y valoración de la cultura propia y } \\
\text { ajena en los elementos patrimoniales. }\end{array}$ \\
\hline & \multirow{6}{*}{$\begin{array}{l}\text { Tipología } \\
\text { patrimonial }\end{array}$} & \multirow{4}{*}{$1 \mathrm{P} 2 \mathrm{P}$} & Natural & Aspectos de carácter medioambiental \\
\hline & & & Histórico & Referentes arqueológicas y documentales \\
\hline & & & Artístico & $\begin{array}{l}\text { Manifestaciones correspondientes a las diferentes } \\
\text { corrientes artísticas }\end{array}$ \\
\hline & & & Etnológico & Elementos tradicionales que explican el cambio social \\
\hline & & \multirow{2}{*}{$\begin{array}{l}1 \mathrm{~A} 2 \mathrm{~A} 3 \mathrm{~A} \\
8 \mathrm{~A}\end{array}$} & $\begin{array}{l}\text { Científico- } \\
\text { Tecnológico. }\end{array}$ & $\begin{array}{l}\text { Elementos industriales y tecnológicos con relevancia } \\
\text { socio-cultural }\end{array}$ \\
\hline & & & Holístico & $\begin{array}{l}\text { Consideración global de todas las manifestaciones } \\
\text { anteriores }\end{array}$ \\
\hline \multirow{5}{*}{ CATEGORÍA II } & \multirow{2}{*}{$\begin{array}{l}\text { Concepto de } \\
\text { ciudadanía }\end{array}$} & $\begin{array}{l}3 \mathrm{P} 4 \mathrm{P} \\
6 \mathrm{P}\end{array}$ & Adaptativa & $\begin{array}{l}\text { Adquisición de comportamientos que permiten estar en } \\
\text { consonancia con el contexto. Confianza en las } \\
\text { instituciones. }\end{array}$ \\
\hline & & 4A 5A 6A & Crítica & $\begin{array}{l}\text { Representada por la participación y el compromiso de } \\
\text { la persona con la sociedad }\end{array}$ \\
\hline & \multirow{3}{*}{$\begin{array}{l}\text { Tipología de } \\
\text { ciudadanía }\end{array}$} & $\begin{array}{l}4 \mathrm{P} 5 \mathrm{P} \\
6 \mathrm{P}\end{array}$ & \multirow[t]{2}{*}{ Pasiva } & \multirow[t]{2}{*}{$\begin{array}{l}\text { Procesos cognitivos de comportamientos cívicos, } \\
\text { funcionamiento y composición de las instituciones y } \\
\text { acciones simples cuyo máximo nivel de compromiso es } \\
\text { la participación electoral }\end{array}$} \\
\hline & & 445 & & \\
\hline & & 4А כА ОА & Participativa & $\begin{array}{l}\text { Implicación y compromiso por mejorar la comunidad a } \\
\text { la que se pertenece }\end{array}$ \\
\hline \multirow{10}{*}{$\begin{array}{l}\text { CATEGORÍA } \\
\text { III }\end{array}$} & \multirow{3}{*}{$\begin{array}{l}\text { Qué } \\
\text { enseñar/ } \\
\text { aprender }\end{array}$} & $5 \mathrm{P} 10 \mathrm{P}$ & Identidad & $\begin{array}{l}\text { Concienciar al alumnado del valor y el respeto del } \\
\text { patrimonio y los rasgos identitarios de la cultura propia } \\
\text { y de las ajenas }\end{array}$ \\
\hline & & \multirow[b]{2}{*}{$5^{\mathrm{a}} \mathrm{A} 10 \mathrm{~A}$} & Conservación & $\begin{array}{l}\text { Importancia de la conservación del patrimonio con una } \\
\text { actitud crítica e intervencionista }\end{array}$ \\
\hline & & & Gestión & $\begin{array}{l}\text { Dotar al alumnado de un pensamiento reflexivo para } \\
\text { interpretar su entorno y actúe responsablemente en el } \\
\text { uso de los elementos patrimoniales }\end{array}$ \\
\hline & \multirow{4}{*}{$\begin{array}{l}\text { Para qué } \\
\text { enseñar/ } \\
\text { aprender }\end{array}$} & \multirow[b]{2}{*}{$5 \mathrm{P}, 10 \mathrm{P}$} & $\begin{array}{l}\text { Visión } \\
\text { culturalista }\end{array}$ & $\begin{array}{l}\text { Conocimiento de hechos históricos e informaciones de } \\
\text { carácter conceptual }\end{array}$ \\
\hline & & & \multirow{2}{*}{$\begin{array}{l}\text { Visión práctico- } \\
\text { conservacionista }\end{array}$} & \multirow[t]{2}{*}{$\begin{array}{l}\text { Importancia de conservar el patrimonio como legado } \\
\text { del pasado para las generaciones futuras }\end{array}$} \\
\hline & & & & \\
\hline & & $8 \mathrm{~A}, 9 \mathrm{~A}$ & $\begin{array}{l}\text { Visión Socio- } \\
\text { crítica }\end{array}$ & $\begin{array}{l}\text { Formación de ciudadanos críticos y activos con el } \\
\text { entorno }\end{array}$ \\
\hline & \multirow{3}{*}{$\begin{array}{l}\text { Cómo } \\
\text { enseñar/ } \\
\text { aprender }\end{array}$} & 7P 8P,9P & $\begin{array}{l}\text { Integración } \\
\text { anecdótica }\end{array}$ & $\begin{array}{l}\text { Actividades puntuales y descontextualizadas sin } \\
\text { relación con el diseño didáctico. }\end{array}$ \\
\hline & & \multirow[b]{2}{*}{$7 \mathrm{~A}$} & $\begin{array}{l}\text { Fuente de } \\
\text { análisis }\end{array}$ & $\begin{array}{l}\text { Uso del patrimonio como recurso para el trabajo e } \\
\text { interpretación del contexto sociohistórico. }\end{array}$ \\
\hline & & & $\begin{array}{l}\text { Imbricación } \\
\text { plena }\end{array}$ & Inclusión plena de los valores cívicos en el patrimonio. \\
\hline
\end{tabular}

En el caso del alumnado, las categorías 1 y 2 son las mismas que las del profesorado, pues la intención es saber el grado de coincidencia o no entre las concepciones de unos y otros, pero 
respecto a la tercera categoría el enfoque cambia, ya que para el alumnado no se trata de enseñar, sino de aprender.

\section{Resultados}

En relación a la primera categoría, los resultados obtenidos nos muestran que la mayoría del profesorado tiene una concepción del patrimonio caracterizada por una visión histórica, en base a la antigüedad de los elementos que lo componen. Por contra, la perspectiva monumentalista es la menos valorada, junto con la monumentalista y simbólico-identitaria.

En el caso de los estudiantes, las perspectivas más valoradas son la monumentalista y fetichista (46\%), en base a criterios de singularidad, escasez, rareza o admiración irracional. Asimismo, un porcentaje muy similar obtiene el patrimonio histórico y estético (45\%).

Por otro lado, respecto a la variable de tipología patrimonial, el total del profesorado tiene una visión holística del patrimonio, considerando la totalidad y variabilidad de los referentes patrimoniales en sus respuestas, aunque con un predominio del patrimonio histórico-artístico.

Los estudiantes, por su parte, tienen una visión del patrimonio incompleta; la inmensa mayoría no llegan a considerar la totalidad y variedad de los referentes patrimoniales, centrándose de forma habitual en las manifestaciones de carácter natural (67\%) e histórico-artístico (64\%), y con una muy baja consideración de manifestaciones de filiación etnológica y tecnológica, y mucho menos, la visión integral y holística del patrimonio.

Sin embargo, hay que destacar que esta subcategoría está muy influenciada por el componente identitario; es decir, si nos fijamos en la primera pregunta, en la que se proponía a los alumnos que se posicionasen acerca de elegir si un elemento de la cultura onubense es patrimonial o no y argumentar sobre dicha elección, el 100\% de los entrevistados marca el Parque Nacional de Doñana como patrimonio, por lo que cabría esperar que la tipología natural esté muy arraigada en sus concepciones. Sin embargo, si vamos a la tercera cuestión, en la que se les da la opción de otros referentes naturales -los desiertos africanos, por ejemplo-, el porcentaje baja (20,7\%), mientras que los referentes patrimoniales relacionados con el fenómeno de Doñana (Lince Ibérico y las marismas de Odiel) siguen obteniendo un porcentaje de representatividad elevado (67\%). Algo muy similar ocurre con el patrimonio industrial, el Muelle de la Compañía de Ríotinto obtiene buena valoración (49 de los 82 alumnos encuestados lo consideran patrimonio) y en la pregunta dos son varios los alumnos que lo nombran en sus respuestas. Lo que cabe preguntarnos es si ante otros ejemplos de patrimonio industrial que no sean símbolos tan conocidos de Huelva, reaccionarían igual.

En relación con el patrimonio etnológico, todo apunta que es el menos valorado, ítems como los botos de Valverde del Camino en la pregunta 1 o la tribu indígena de los Papúas de Nueva Guinea en la pregunta tres, obtienen porcentajes poco representativos, aunque se produce una excepción con el jamón ibérico, marcado por 47 alumnos; sin embargo, de estos sólo 45 dan una respuesta razonada del porqué, lo que evidencia que la valoración que tiene la sociedad de consumo del patrimonio influye en el alumnado.

Con respecto a la categoría dos: concepciones de ciudadanía. Es interesante destacar que los profesores se posicionan dentro de una ciudadanía crítica, puesto que la inmensa mayoría de los encuestados se define por considerar que la conservación del patrimonio es una obligación conjunta de instituciones públicas y ciudadanos $(77,7 \%$ ), aunque no se plantean medidas concretas a través de las cuales se pueda llevar a cabo tal conservación y gestión desde posturas activas y comprometidas. Sólo 1 de los 9 profesores participantes explica un proyecto práctico a nivel europeo propio de una ciudadanía crítica. 
Esta tendencia se confirma en la pregunta cinco, cuando les preguntamos que de qué manera las Ciencias Sociales y el patrimonio pueden ser valiosas para la formación de ciudadanos, nuevamente las respuestas se posicionan en la necesaria implicación de la sociedad en labores como la conservación y gestión de los bienes, pero si les preguntamos por acciones concretas mediante las cuales puedan implicar a su alumnado y formarles en tal propósito, comprobamos que todas estas declaraciones nuevamente se quedan en un plano teórico.

En el caso del alumnado, el concepto de ciudadanía predominante es el de ciudadanía adaptativa, donde prima la confianza en las instituciones; ya sean públicas, como la Junta de Andalucía, que obtiene el 30,4\% del total de respuestas; o privadas, como asociaciones encargadas de la conservación del patrimonio, con un porcentaje de 30\%. Llama la atención que sólo un 2\% crea que desde su entorno educativo pueda ejercer comportamientos propios de una ciudadanía activa y crítica, tendencia que se confirma en la pregunta cinco, en la que 11 de los 82 alumnos entrevistados consideran que los ciudadanos no podemos hacer nada ante una destrucción injusta de un elemento patrimonial de reconocido prestigio. Sin embargo, si pasamos a la segunda subcategoría referida a la tipología, comprobamos que el 48,7\% de los estudiantes encuestados entienden las manifestaciones legales como una acción fuertemente vinculada al tipo de ciudadanía, enmarcándose dentro de una ciudadanía participativa y, cuando les pedimos que propusieran ellos acciones ciudadanas (pregunta 5), el $60,9 \%$ de las respuestas apuntaban a comportamientos que caracterizan a una ciudadanía participativa.

Por último, en lo referente a la categoría tres: Concepciones sobre la E/A de la ciudadanía a través del patrimonio.

La primera de las subcategorías: qué enseñar/qué aprender; nos muestra que los profesores entienden que tienen que enseñar identidad, conservación y gestión. Los tres indicadores han recibido puntuaciones altas y similares. Sin embargo, en el caso de los alumnos, identidad y conservación son los descriptores que tienen porcentajes más elevados.

El dato más destacable es que el $85 \%$ de los alumnos encuestados se muestra favorable a la pregunta de si creen que de alguna manera el patrimonio te puede enseñar a ser ciudadano, aunque el 14.6\% de ellos no sabe cómo y la mayoría de respuestas está relacionada con actitudes o valoraciones morales, refiriéndose a su protección y conservación como símbolos de un pasado que no debe perderse.

En el caso de la subcategoría cómo enseñar/cómo aprender, domina entre los profesores una concepción de la didáctica del patrimonio y la ciudadanía en la que prima la integración plena con los contenidos de la materia de Ciencias Sociales. El 93,3\% cree que la mejor forma de integrar el patrimonio en la enseñanza de la ciudadanía es diseñando actividades complementarias en las que se trabajen los conceptos de identidad, conservación y gestión del patrimonio, seguido de la opción "a través de la integración en los programas docentes de los componentes conceptuales, procedimentales y actitudinales" (88,8\%). Los datos obtenidos de las preguntas 8 y 9 matizan estos porcentajes, puesto que puntuaciones similares obtienen las respuestas "como eje en torno al cual se diseñan unidades didácticas para el trabajo en el aula" (60\%), "como complemento a las actividades y propuestas recogidas en los libros de texto" (55,5\%), "como actividades esporádicas" (55,5\%). En la pregunta 8 comprobamos que el mayor grado de representatividad de la muestra entiende los referentes patrimoniales como fuentes de información complementaria a los contenidos tratados en el aula: "la visita a algún centro de interpretación y conservación del patrimonio", "el análisis de elementos patrimoniales propios de tu ciudad".

Para los alumnos, la mejor forma de aprender ciudadanía a través del patrimonio es realizando visitas fuera del aula $(24,6 \%)$, así como llevando a cabo algún proyecto para proteger un elemento patrimonial en peligro de su ciudad (20\%). En este caso, comprobamos que las concepciones de profesores y alumnos con respecto a esta subcategoría son parejas, dominando en 
ambos casos una integración plena de la didáctica del patrimonio y de la ciudadanía en los procesos de enseñanza/aprendizaje de las Ciencias Sociales. Sin embargo, a falta de un estudio en mayor profundidad, podemos intuir que las respuestas dadas por el profesorado a este bloque de preguntas está más en el plano declarativo que en su práctica de aula, debido a la ambigüedad que muestra en algunas cuestiones y al porcentaje de alumnos (18\%) que escogen respuestas relacionadas con una visión unidireccional de los métodos de aprendizaje, como "la charla de un político", "la explicación de un profesor", "la charla de un director de museo o centro de interpretación". Esto nos lleva a pensar que en el contexto escolar siguen primando los métodos tradicionales de enseñanza/aprendizaje, aunque docentes y discentes crean que no son los más acertados para conseguir aprendizajes significativos. Los datos obtenidos invitan a la reflexión y a preguntarnos qué clase de formación se imparte en nuestras aulas en la que los dos actores protagonistas no están de acuerdo con los métodos utilizados.

En lo que respecta a la subcategoría para qué enseñar/para qué aprender, destaca la tendencia a considerar el patrimonio como un instrumento para la formación de ciudadanos críticos y activos con el entorno, para lo cual es necesario la adquisición de unos contenidos a través de los cuales los estudiantes podrán entender y valorar los elementos patrimoniales y adquirir los comportamientos ciudadanos que les lleven a ejercer la conservación y gestión de los mismos. Así, comprobamos que los profesores tienen una visión culturalista, práctico-conservacionista y sociocrítica del patrimonio, constituyendo un instrumento de primer orden para la educación de una ciudadanía activa. Sin embargo, estas concepciones de los profesores son totalmente opuestas a las concepciones de los alumnos. La gran mayoría de los discentes encuestados dan respuestas relacionadas con la adquisición o acumulación de datos o informaciones de carácter conceptual (77,4\%), igualmente, llama la atención que ninguno de ellos considere la necesidad de valorar el pasado y aprender a conservarlo para generaciones futuras, y la escasa significatividad que obtiene la visión socio-crítica $(2 \%)$. Hay que destacar también, el porcentaje de alumnos que mencionan actividades relacionadas con el ocio y el deporte $(7,6 \%)$ y los que no contestan a la pregunta $(16 \%)$, que puede no tengan interés en aprender.

\section{Conclusiones}

Conforme a las preguntas que nos planteábamos al inicio de nuestra investigación, podemos extraer las siguientes conclusiones.

Los profesores manifiestan una visión global e integrada del patrimonio, no sólo en términos de reconocer un abanico amplio de tipología patrimonial, sino también a la hora de establecer relaciones o conexiones entre los recursos naturales, económicos, sociales, culturales e históricos, pero no llegan a desarrollar una perspectiva simbólico-identitaria de los referentes patrimoniales de forma que se integre en todos los elementos los aspectos culturales que puedan definir a una sociedad. Además, comprobamos que a pesar de considerar la totalidad y variabilidad de los referentes patrimoniales, hay en ellos una predominancia de las manifestaciones de carácter histórico-artístico.

En el caso de los alumnos, presentan ciertas dificultades de valoración de algunas tipologías patrimoniales, como el patrimonio etnográfico. Sin embargo, el patrimonio natural es tan valorado como el histórico-artístico; el segundo no es de extrañar, debido a las concepciones que tienen los profesores sobre él y a que ha sido tratado tradicionalmente en la cultura académica. En el caso del patrimonio natural, creemos que es tan valorado por los estudiantes porque conecta mucho más con la sensibilidad de los alumnos, suponemos que por la difusión en la sociedad occidental de los valores ecológicos, y especialmente en el caso de Huelva, por los problemas medioambientales 
causados por la contaminación. Pero es necesario realizar una puntualización a este respecto, ¿esta valoración del patrimonio natural es real o debemos relacionarla con una perspectiva fetichista influenciada por los medios? Y en el caso de que fuera real, ¿'los alumnos valorarían otros bienes naturales que no fueran de Huelva?

Si pasamos a las concepciones de ciudadanía, los datos obtenidos ponen de manifiesto que para la mayoría del profesorado, el aprendizaje del patrimonio ayuda a conocer la organización política y social del grupo y el pasado para entender o justificar el presente. Piensan que fomenta el civismo, el respeto y la tolerancia. También opinan que la educación ciudadana ayuda a la formación de opiniones, a tener criterios para las mismas, a emitir juicios y a ser críticos. Igualmente ayudan a formar ciudadanos democráticos y sirven para entender el multiculturalismo. Pero por otro lado, podemos decir que los responsables de formar a los ciudadanos del presente más inmediato y del futuro encuentran dificultades a la hora de trasladar estas ideas a su práctica, pues no han hecho ninguna referencia a acciones educativas concretas.

Así, el alumnado tiene en general un concepto de ciudadanía muy limitado. Llama la atención que sólo un $2 \%$ de los alumnos encuestados ven en la comunidad educativa y en el contexto escolar la confianza para implicarse activamente en la gestión y defensa de sus intereses; por el contrario, la gran mayoría eligen instituciones públicas o privadas. Respecto a las formas de acción ciudadana conocidas por el alumnado, la respuesta más recurrente es "manifestación, huelga o protesta". Sin embargo, no hemos encontrado ninguna respuesta que aluda a la creación de una asociación o plataforma ciudadana, ni a la participación pública en plenos del ayuntamiento o redacción de alegaciones y recursos. Esto nos hace pensar que estas vías son desconocidas por el alumnado, probablemente porque este conocimiento no se enseña en la escuela y el alumnado lo suple a través de los medios de comunicación, por eso los únicos comportamientos ciudadanos que obtenemos en sus respuestas son los recogidos por los medios.

Por último, es interesante destacar que la mayoría de los encuestados considera que el aprendizaje del patrimonio y la ciudadanía tiene como finalidad la adquisición o acumulación de datos o informaciones de carácter conceptual. Creemos que ello es debido a un proceso de enseñanza/aprendizaje ligado a los métodos tradicionales de enseñanza, donde prima el conocimiento conceptual de los elementos patrimoniales, dejando poco margen a los componentes actitudinales, que son tan importantes para poder adquirir una educación como ciudadano. Además, en los resultados de la pregunta ocho formulada a los docentes y relacionada con las actividades, la mayor parte de las respuestas se centran en visitar elementos patrimoniales o el análisis de uno de ellos, lo que indica una tendencia a considerar el patrimonio como fuente o recurso de los contenidos tratados en el aula, confirmando nuestra hipótesis acerca de que la práctica de aula responde al modelo didáctico tradicional.

Pero lo más destacable de nuestro estudio es que los alumnos encuestados se muestran interesados en participar en propuestas de comunicación recíproca en torno a elementos patrimoniales con el fin de adquirir comportamientos cívicos y democráticos. A pesar de recibir una educación patrimonial de carácter conceptual y una formación ciudadana pasiva y adaptativa, sí que ven en los referentes patrimoniales un instrumento educativo de primer orden para llegar a obtener un pensamiento crítico que les lleve a tener un comportamiento activo y comprometido con el entorno.

Si a esto unimos las concepciones del profesorado que, como hemos podido apreciar, ven el patrimonio como un instrumento educativo de primer orden, creemos que es necesario llevar a cabo investigaciones en mayor profundidad que nos revelen las dificultades u obstáculos que los profesores encuentran a la hora de poner en práctica estas ideas. 


\section{Referencias}

Ávila, R. M. (2.001). Historia del Arte, enseñanza y profesores. Sevilla: Díada.

Ávila, R. M. (2.005). Reflexiones sobre la enseñanza y aprendizaje del patrimonio integrado. Una experiencia en la formación de maestros. Investigación en la Escuela, 56, 43-54.

ÁVILA, R. M. y MATOZZI, I. (2.009). La didáctica del patrimonio y la educación para la ciudadanía. En R.M. Ávila, B. Borghi e I. Matozzi (Eds.), L' educazione alla cittadinanza europea e la formazione degli insegmanti (pp. 327-352). Bolonia: Pàtron editore.

Ávila, R. M, y Duarte, O. (2012). Salvemos el patrimonio, patrimonio en peligro. Una actividad para la formación docente y ciudadana. En N. de Alba, F. F. García Pérez, y A. Santisteban (Eds.), Educar para la participación ciudadana en la enseñanza de las ciencias sociales (pp.471-480). Sevilla: Díada Editora

Cuenca, J.M. (2004). El patrimonio en la Didáctica de las Ciencias Sociales. Análisis de concepciones, dificultades y obstáculos para su integración en la enseñanza obligatoria. Michigan: Proquest-Universidad de Michigan. Rescatado de http://wwwlib.umi.com/cr/uhu/fullcit?p3126904

Cuenca, J. M. (2.010). El Patrimonio en la didáctica de las Ciencias Sociales: análisis de concepciones, dificultades y obstáculos para su integración en la enseñanza obligatoria. Universidad de Huelva: Servicio de Publicaciones.

Cuenca, J.M. y Martín, M. (2.009). La formación del profesorado para formar ciudadanos: el papel de la educación patrimonial. En R.M. Ávila, B. Borghi e I. Matozzi (Eds.) L'educazione alla cittadinanza europea e la formazione degli insegmanti (pp. 507-51). Bolonia: Pàtron editore.

Domínguez, C. y Cuenca, J.M. (2.005). Patrimonio e identidad para un espacio educativo multicultural. Análisis de concepciones y propuesta didáctica. Investigación en la Escuela, 56, $27-42$.

Estepa, J. (2.009). La educación del patrimonio y la ciudadanía europea en el contexto español. En R.M. Ávila, B. Borghi e I. Matozzi (ed.) L'educazione alla cittadinanza europea e la formazione degli insegmanti (pp. 353-362). Bolonia: Pàtron editore.

Estepa, J. y Morón, $\mathrm{M}^{\mathrm{a}}$ C. (2013). La educación patrimonial en los materiales didácticos: la visión del profesorado de Ciencias Sociales, Geografía e Historia. En J. Estepa, (Ed.), La educación patrimonial en la escuela y el museo: investigación y experiencias (pp.145-165). Universidad de Huelva: Servicio de Publicaciones.

Estepa, J.; Wamba, A. M. y Jiménez, R. (2005). Fundamentos para una enseñanza y difusión del patrimonio desde una perspectiva integradora de las ciencias sociales y experimentales. Investigación en la Escuela, 56, 19-26.

Estepa, J. y Ávila, R.M. (2007). Concepciones sobre la enseñanza y difusión del Patrimonio en las instituciones educativas y en los centros de interpretación. Estudio descriptivo. Enseñanza de las Ciencias Sociales, 6, 75-94.

Estepa, J.; Ávila, R. M. y Ferreras, M. (2008). Primary and secondary teachers' conceptions about heritage and heritage education: A comparative analysis. Teaching and Teacher Education, 24, 2095-2107.

Estepa, J., Ferrreras, M., y Morón, M.C. (2013). Resultados de investigación sobre concepciones del profesorado y gestores del patrimonio y análisis de libros de texto y materiales didácticos de los museos y centros de interpretación del patrimonio. En J. Estepa (Ed.), La Educación patrimonial en la escuela y el museo: investigación y experiencias (pp. 25-40). Universidad de Huelva: Servicio de Publicaciones. 
Gil-Díez, I. (2012). Heredar y gobernar. Una propuesta de educación de la participación ciudadana a través de la didáctica del patrimonio. En N. De Alba, F.F. García y A. Santisteban (Eds.), Educar para la participación ciudadana en la enseñanza de las ciencias Sociales (pp. 537-542). Sevilla. Díada Editora.

Martín, M., Cuenca, J. M., y Bedía, J. (2012). Educación para la participación ciudadana a través del patrimonio: experiencias en el museo de Huelva. En N. De Alba, F.F. García y A. Santisteban (Eds.), Educar para la participación ciudadana en la enseñanza de las ciencias Sociales (pp.19-26). Sevilla: Díada Editora

Rodríguez, G., Gil, J., y García, E. (1996). Metodología de la investigación cualitativa. México: Ediciones Aljibe.

Texeira, S. (2006). Educación patrimonial: alfabetización cultural para la ciudadanía. Estudios Pedagógicos, 32, 133-145.

Travé, G., Pozuelos, F. J., Cañal, P., y de Las Heras, M. Á. (2013). Experimentación de una guía de análisis de materiales y desarrollo de la enseñanza del medio natural y social. Investigación en la Escuela, 81, 5-20.

\section{Sobre los autores}

Autoa: Laura Lucas Palacios

Institución: Universidad de Valladolid

E-mail: laura.lucas.palacios@uva.es

Información biográfica: Profesora de la Facultad de Educación de Palencia, dentro del Departamento de Didáctica de las Ciencias Sociales. Miembro del grupo de investigación DESYM "Formación Inicial y Desarrollo Profesional del Profesorado" y de Red 14. Línea principal de investigación: Educación Patrimonial y Educación para la ciudadanía en la Didáctica de las Ciencias Sociales.

ORCID: no disponible

Autor: Jesús Estepa Giménez

Institución: Universidad de Huelva

E-mail: jestepa@uhu.es

Información biográfica: Catedrático del Cuerpo de "Profesores de Enseñanza Secundaria", especialidad "Geografía e Historia" y Catedrático de Universidad del Área de Didáctica de las Ciencias Sociales, en el Departamento de Didácticas Integradas de la Universidad de Huelva, desde julio de 2004. Miembro del grupo de investigación DESYM "Formación Inicial y Desarrollo Profesional del Profesorado" (HUM 168 del Plan Andaluz de Investigación de la Junta de Andalucía), de la Red IRES y la Red 14. Líneas principales de investigación: 1) Comunicación y Educación Patrimonial 2) Didáctica de la Historia y Ciudadanía 3) Investigación escolar en la enseñanza de las Ciencias Sociales

ORCID: http://orcid.org/0000-0002-6381-8331 


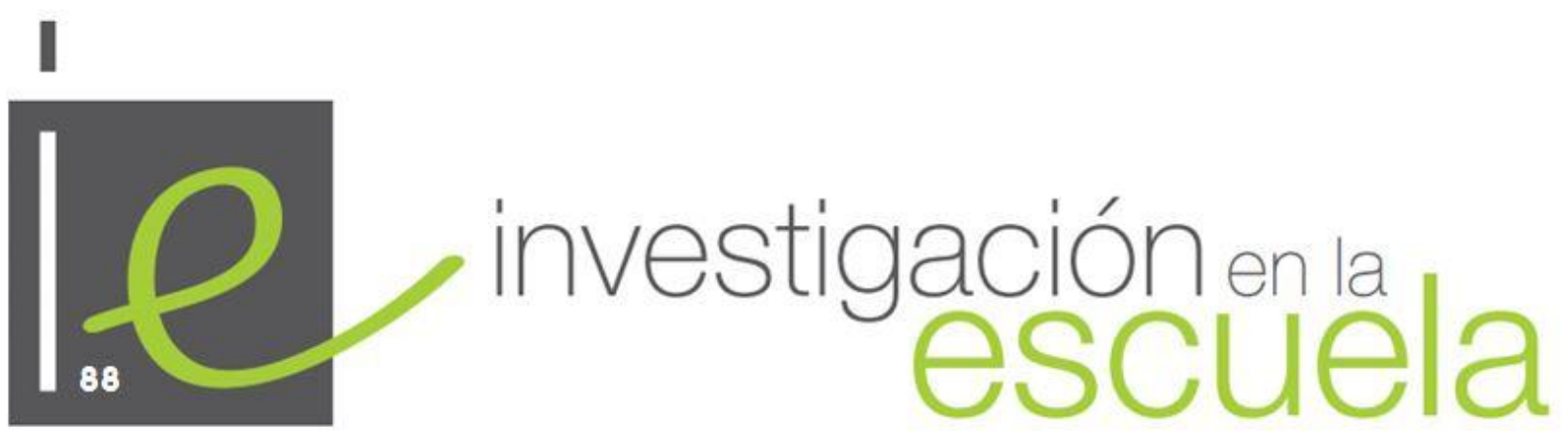

Revista académica evaluada por pares y de acceso abierto

Número 89

15 de noviembre de 2016

ISSN 2443-9991

\section{(c)}

SOMERIIGHISRESERVED LOs/as lectores/as pueden copiar, mostrar, y distribuir este artículo, siempre y cuando se de crédito y atribución al autor/es y a Investigación en la Escuela, se distribuya con propósitos no-comerciales, no se altere o transforme el trabajo original. Más detalles de la licencia de Creative Commons se encuentran en http://creativecommons.org/licenses/by-nc-sa/3.0 Cualquier otro uso debe ser aprobado en conjunto por el autor/es, o Investigación en la Escuela.

Contribuya con comentarios y sugerencias en la web de la revista. Por errores y sugerencias contacte a investigacionescuela@,ddcc.uhu.es 


\title{
Investigación en la escuela
}

Consejo de dirección: Ana Rivero García (Universidad de Sevilla), Nicolás de Alba Fernández (Universidad de Sevilla), Pedro Cañal de León (Universidad de Sevilla), Francisco F. García Pérez (Universidad de Sevilla), Gabriel Travé González, (Universidad de Huelva), Francisco F. Pozuelos Estrada (Universidad de Huelva)

\author{
Dirección: Ana Rivero García y Nicolás de Alba Fernández \\ Técnico de edición: Francisco Javier López Sánchez
}

\section{Consejo editorial}

José Félix Angulo Rasco. Universidad de Cádiz Rosa Ma Ávila Ruiz. Universidad de Sevilla Pilar Azcárate Goded. Universidad de Cádiz Juan Bautista Martínez Rodríguez. Universidad de Granada

Nieves Blanco García. Universidad de Málaga Fernando Barragán Medero. Universidad de La Laguna José Carrillo Yáñez. Universidad de Huelva José Contreras Domingo. Universidad de Barcelona. Luis C. Contreras González. Universidad de Huelva Ana $\mathbf{M}^{\mathbf{a}}$ Criado García-Legaz. Universidad de Sevilla Rosario Cubero Pérez. Universidad de Sevilla José $\mathbf{M}^{\mathbf{a}}$ Cuenca López. Universidad de Huelva Jesús Estepa Giménez. Universidad de Huelva Rafael Feito Alonso. Universidad Complutense (Madrid)

Francisco José García Gallardo. Universidad de Huelva

Soledad García Gómez. Universidad de Sevilla J. Eduardo García Díaz. Universidad de Sevilla
Fernando Hernández Hernández. Universidad de Barcelona

Salvador Llinares Ciscar. Universidad de Alicante Alfonso Luque Lozano. Universidad de Sevilla Rosa Martín del Pozo. Universidad Complutense (Madrid)

José Martín Toscano. IES Fernando Herrera (Sevilla) Jaume Martínez Bonafé. Universidad de Valencia F. Javier Merchán Iglesias. Universidad de Sevilla Emilia Moreno Sánchez. Universidad de Huelva. Rosario Ortega Ruiz. Universidad de Córdoba Antonio de Pro Bueno. Universidad de Murcia Fco. de Paula Rodríguez Miranda. Universidad de Huelva

Pedro Sáenz-López Buñuel. Universidad de Huelva Antoni Santisteban Fernández. Universidad Autónoma (Barcelona)

Emilio Solís Ramírez. Catedrático de IES.

$\mathbf{M}^{\mathbf{a}}$ Victoria Sánchez García. Universidad de Sevilla. Magdalena Suárez Ortega. Universidad de Sevilla

\section{Consejo asesor}

Manuel Area Moreira. Universidad de La Laguna

Jaume Carbonell. Director Cuadernos de Pedagogía. Barcelona

César Coll. Universidad de Barcelona

Christopher Day. Universidad de Nothingham. U.K.

Juan Delval. Universidad Nacional de Educación a Distancia

John Elliott. Universidad de East Anglia. Norwich. U.K.

José Gimeno Sacritán. Universidad de Valencia

André Giordan. Universidad de Paris VII y Ginebra

Francisco Imbernón. Universidad de Barcelona

Ángel Pérez Gómez. Universidad de Málaga

Rafael Porlán Ariza. Universidad de Sevilla

Francesco Tonucci. Instituto de Pedagogía del C.N.R. Roma

Jurjo Torres Santomé. Universidad de A Coruña 
